\title{
Postpartum bullous pemphigoid - A case report
}

\author{
Malar Nisha Raveendran, Sundaramoorthy M. Srinivasan
}

\author{
Department of Dermatology and STD, Chettinad Hospitals and Research Institute, Kelambakkam, Tamil Nadu, India
}

Corresponding author: Prof. Sundaramoorthy M. Srinivasan, E-mail: hamsrini@yahoo.co.in

\begin{abstract}
Bullous pemphigoid (BP) is an acquired autoimmune blistering disease occurring in the elderly more than 70 years of age though may occur during young age and childhood. BP is categorized by the development of urticarial plaques surmounted by sub epidermal blisters, and the deposition of immunoglobulin and complement at the basement membrane zone (BMZ) of the skin. BP is associated with other diseases, such as diabetes mellitus, thyroid disorders, rheumatoid arthritis and malignancies. Heat shock proteins (HSP) are a family of proteins that are produced by cells in response to exposure to stressful conditions. Heat shock protein 90 (HSP90) inhibitors potentially target for the autoimmune disease and chronic inflammatory diseases. We present a case of 21 year old lactating female, a known case of hyperthyroidism with an abrupt onset of fluid filled vesicles and bullae over the abdomen and limbs since 3 days associated with itching. She had similar episodes before and was diagnosed and treated for BP. She had a flare up of the disease due to the personal stress in the patient as Heat shock proteins plays an important role in the pathophysiology of BP. Following treatment upon continuous follow ups, there were no flare up of the disease. Here in this patient, apart from hypothyroidism, stress also acts as a trigger in BP.
\end{abstract}

Key words: Bullous pemphigoid; Pregnancy; Heat shock proteins; Immune modulation

\section{INTRODUCTION}

Bullous pemphigoid (BP) is an acquired autoimmune blistering disorder occurring in the elderly individuals more than 70 years of age [1]. It is also reported in young age and during infancy and childhood [2]. The incidence in people older than 80 years is $150-180$ per million per year [1]. BP is the most common bullous disease in Western Europe than in India [2].

Heat shock proteins (HSP) are a family of proteins that are produced by cells in response to exposure to stressful conditions. They were first described in relation to heat shock [3]. Hence the name. But are now known to also be expressed during other stresses including exposure to cold [4], UV light [5], and during wound healing or tissue remodelling. The heat shock proteins are also referred to as stress proteins and their up regulation is sometimes described more generally as part of the stress response [6] .

\section{CASE REPORT}

A 21 year old lactating female, married since one year presented to us with an abrupt onset of fluid filled vesicles and bulla over the abdomen and limbs since 3 days associated with itching with few erosions. She presented with history suggestive of wheal formation and severe itching prior to the development of lesions. No history of spontaneous rupture of lesions, peripheral extension, mucosal lesions or history suggestive of healing with milia formation. No history of prior drug intake, or any constitutional symptoms, systemic involvement in the patient. Patient gave history of personal stress following her delivery. She's a known case of hypothyroidism and is on continuous treatment with T. Eltroxin $25 \mathrm{mcg}$ for 4 years. Two years back, patient had similar episodes following stressful events and was on treatment with systemic corticosteroids. No flare up of the disease was seen in these two years. Elective LSCS done on 23-09-2015, delivered a healthy girl baby weighing $2.5 \mathrm{~kg}$.

Cutaneous examination revealed multiple tense vesicles and bullae with clear filled fluid over an erythematous base predominantly over the flexural aspects of upper limb, medial aspects of thighs and few over the trunk and abdomen interspersed with few erosions and crusting (Figs. 1 and 2). Oral cavity 
and genital examination were normal. Nikolsky's sign was negative and Bullae spreading sign was positive. Elsewhere the skin was normal. Her baby was healthy and did not show any skin lesions. A differential diagnosis of bullous pemphigoid was considered.

Laboratory investigations were done; complete blood analysis revealed an eosinophil count of $9.8 \%$. Histopathological investigation of a fresh intact bullae showed sub epidermal cleft with mixed infiltrate of eosinophils and neutrophils (Fig. 3). Based on the clinical and histopathology finding a diagnosis of BP was confirmed.

Pediatrician advice was sought regarding the baby's condition which was normal and suggested to continue breast feeding as steroids are under category B which will not be harmful to the baby. General physician advised to continue T. Eltroxin $25 \mathrm{mcg}$ for the patient's hypothyroid status.

Patient was treated with oral prednisolone lmg per kg body weight, to which she had a dramatic response. Patient improved symptomatically and was discharged after two weeks. She has been under regular follow up for past 8 months and asymptomatic till now.

\section{DISCUSSION}

$\mathrm{BP}$ is an increasingly common immunobullous disease of the elderly, and, due to the late age of onset, is rarely seen in women of fertile age [7]. Mucous membrane involvement may be seen in 10\%-30\% of patients [2]. The term BP was coined by Lever in 1953 [8]. Women and men are equally afflicted.

The etiology of BP is idiopathic. Genetic susceptibility shows a strong association with HLA-DQB1 *0301 [9]. Precipitating factors include influenza vaccination, UV light, radiotherapy for breast cancer, thermal and electric burns, percutaneous endoscopic gastrostomy, amputation stump, incisional hernia scar, and injection or an adhesive dressing [10]. Certain infections like cytomegalovirus, Ebstein-Barr virus, HHV-6, HHV-8, hepatitis B and C viruses, Helicobacter Pylori and Toxoplasma Gondii [2,9] and certain drugs like furosemide, enalapril, phenacitin, ibuprofen, sulfa pyridine also induces BP [10]. Stress also plays as a trigger in the manifestation of $\mathrm{BP}[11]$.

The pathogenesis of BP is characterized by tissuebound and circulating $\operatorname{IgG}$ autoantibodies against

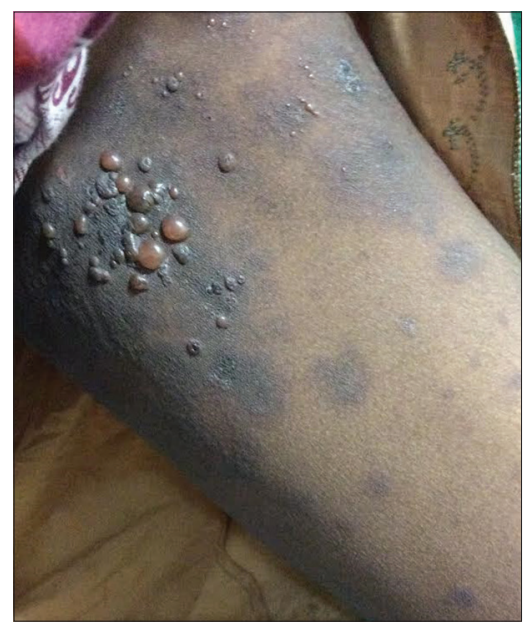

Figure 1: Clinical picture showing tense vesicles and bullae with crusting with surrounding areas of hyperpigmentation on the medial aspect of the left thigh.

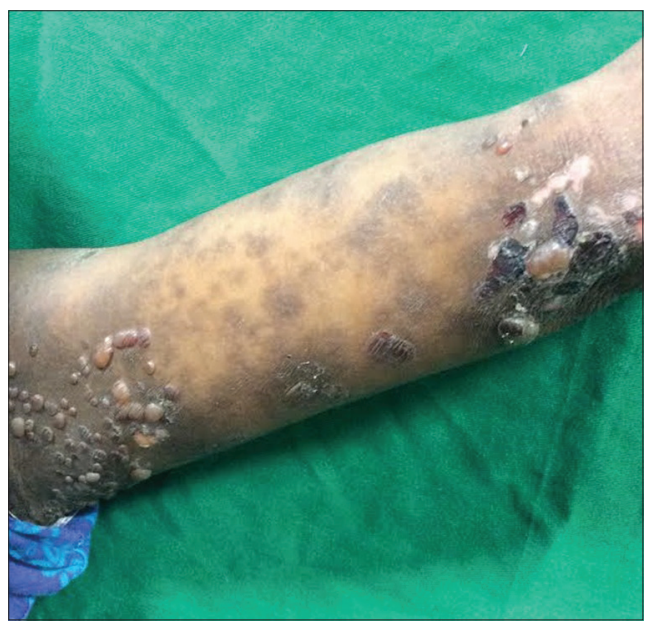

Figure 2: Clinical picture showing vesicles and bullae with crusting on the left upper limb.

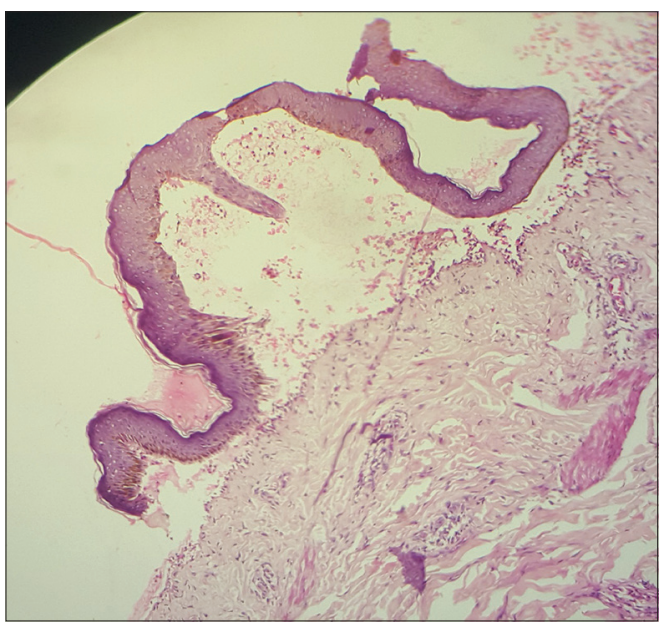

Figure 3: Histopathology showing sub epidermal cleft with mixed infilterate of eosinophils and neutrophils.

two components of the hemi desmosome of stratified epithelia, BP $230 \mathrm{kD}$ (BPAgl) and BP $180 \mathrm{kD}$ 
(BPAg2) [2]. BP manifests as tense blisters on urticarial base more commonly in the lower abdomen, thighs and forearms although they may occur anywhere. Pruritus is present and may be persist for several months. The blister ruptures to leave erosions which heal rapidly with post inflammatory hyperpigmentation.

Histopathology shows sub epidermal clefts and dermal inflammatory infiltrate composed predominantly of eosinophil and neutrophils [1].

$\mathrm{BP}$ is associated with other diseases, such as diabetes mellitus, thyroid disorders, rheumatoid arthritis, pemphigus foliaceus, dermatomyositis, ulcerative colitis, multiple autoimmune diseases, myasthenia gravis and malignancies [10]. As per the 2012 BAD guidelines, both systemic and topical corticosteroids and anti-inflammatory antibiotics are given for mild and moderate cases. In cases of severity, steroid sparing agents like azathioprine, methotrexate, dapsone, $\mathrm{MMF}$ and chlorambucil are given [12].

In 2001 Gee BC et al described BP in two patients that have differed in the disease behaviour during pregnancy [7]. This patient had her first episode 2 years before pregnancy, she was totally asymptomatic during pregnancy, now the patient had a flare up 3 months after pregnancy.

Heat-shock proteins are named according to their molecular weight [13]. Heat shock protein 90 (HSP90) is involved in conformational and structural maturation of signalling molecules and transcription factors in immune reaction. HSP90 inhibitors induce immune modulation via anti-inflammatory effect, regulating humoral and cellular immune responses. Therefore, HSP90 inhibitors potentially useful target for the autoimmune disease and chronic inflammatory diseases. In 2015, studies performed by Stephan Tukaj et al provide an important insight that Hsp90 were highly expressed in the epidermis of BP patients, but its serum levels were decreased and inversely associated with autoantibodies against BP180 NC16A. It may be speculated that anti-BP180 NCl6A autoantibodies indirectly lead to an enhanced intracellular expression of Hsp90 by generation of an inflammatory response comprising soluble pro-inflammatory mediators (cytokines and ROS) and simultaneously cause a direct inhibition of its cellular release $[14,15]$.

In the above discussed case, patient had her first episode 2 years before pregnancy due to the stressful events, she was totally asymptomatic during pregnancy, now the patient had a flare up 3 months after pregnancy. She gave a history of personal stress after delivery following which she developed a flare up of the disease. Due to the stress and hypothyroidism in this patient, she developed BP during the postpartum and not during pregnancy because of the maintained guarded therapy during her initial episode. Upon follow up visits, there were no flare up of the disease as the stress reduced and the thyroid levels were maintained.

\section{CONCLUSION}

$\mathrm{BP}$ is the most common autoimmune blistering disorder occurring in the elderly individuals more than 70 years of age. As per the literature, BP among young people is more severe and more active than the usual form in the elderly. Autoantibodies to stress proteins like Hsp90 also plays an important role in the pathogenesis of BP.

\section{REFERENCES}

1. Di Zenzo G, Della Torre R, Zambruno G, Borradori L, Bullous Pemphigid: from the clinic to the bench. Clin Dermatol. 2012;30:3-16.

2. Wojnarowska F, Venning VA. Immunobullous diseases. In: Burns T, Breathnach S, Cox N, Griffiths C, editors, Rook's Textbook of dermatology. 8th ed. Chichester: Wiley-Blackwell; 2010 p. 40.24-62.

3. Ritossa F. A new puffing pattern induced by temperature shock and DNP in drosophila. Experientia. 1962;18:571-3.

4. Matz JM, Blake MJ, Tatelman HM, Lavoi KP, Holbrook NJ. Characterization and regulation of cold-induced heat shock protein expression in mouse brown adipose tissue. Am J Physiol. 1995;269:38-47.

5. Cao Y, Ohwatari N, Matsumoto T, Kosaka M, Ohtsuru A, Yamashita S. TGF- $\beta 11$ mediates $70-\mathrm{kD}$ a heat shock protein induction due to ultraviolet irradiation in human skin fibroblasts. Pflugers Arch. 1999;438:239-44.

6. Santoro MG. Heat shock factors and the control of the stress response. Biochem Pharmacol. 2000;59:55-63.

7. Gee BC, Allen J, Khumalo NP, Wojnarowska F. Bullous pemphigoid in pregnancy: contrasting behaviour in two patients. Br J Dermatol. 2001;145:994-7.

8. Lo Shiavo A, Ruocco E, Brancaccio G, Caccavale S, Ruocco V, Wolf R. Bullous pemphigoid: Etiology, pathogenesis and inducing factors: Facts and controversies. Clinic Dermatol. 2013;31:391-9.

9. Nozu T, Mita H. Bullous pemphigoid and percutaneous endoscopic gastrostomy. Inter Med. 2010;49:971-5.

10. Khandpur S, Verma P. Bullous pemphigoid. Indian J Dermatol Venereol Leprol. 2011;77:450-5.

11. Cunha PR, Barraviera SR. Autoimmune bullous dermatoses. An Bras Dermatol. 2009;84:111-24.

12. Venning VA, Taghipour K, Mohd Mustapa MF, Hghet AS, Kirtschig G. British Association of Dermatologists' guidelines for the management of bullous pemphigoid 2012. Br J Dermatol. 2012;167:1200-14.

13. Li Z. Srivastava P. Heat-Shock Proteins. Current Protocols in Immunology. 20114;58:1T: A.1T.1-A.1T.

14. Tukaj S, Zillikens D, Kasperkiewicz M. Heat shock protein 90: a 
www.odermatol.com

pathophysiological factor and novel treatment target in autoimmune bullous skin diseases. Exp Dermatol.2015;24:567-71.

15. Tukaj S, Kleszczyński K, Vafia K, Groth S, Meyersburg D, Trzonkowski P, et al. Aberrant Expression and Secretion of Heat Shock Protein 90 in Patients with Bullous Pemphigoid. Cominelli F, ed. PLoS ONE. 2013;8:e70496.
Copyright by Malar Nisha Raveendran, et al. This is an open access article distributed under the terms of the Creative Commons Attribution License, which permits unrestricted use, distribution, and reproduction in any medium, provided the original author and source are credited.

Source of Support: Nil, Conflict of Interest: None declared. 